\title{
CCD photometry of distant open clusters
}

\section{Berkeley 18}

\author{
J. Kaluzny ${ }^{\star}$ \\ Warsaw University Observatory, Al. Ujazdowskie 4, 00-478 Warsaw, Poland \\ e-mail: jka@sirius.astrouw.edu.pl
}

Received April 11; accepted June 11, 1996

\begin{abstract}
We present CCD BVI photometry for Berkeley 18, a rich open cluster located in the Galactic anticenter. The cluster age is similar to, or mariginally lower than age of $\mathrm{M} 67$. Its distance is estimated at $5.8 \mathrm{kpc}$ and the reddening $E(B-V)$ is probably higher than 0.46 . Our data indicate that Be 18 is one of the most populous objects in the whole sample of known galactic open clusters. The angular diameter of the cluster is estimated at 26 arcmin, which corresponds to a linear size of about $44 \mathrm{pc}$. The horizontal branch of Be 18 exhibits a large width in comparison with other old open clusters. ${ }^{1}$
\end{abstract}

Key words: open clusters and associations: individual: Berkeley 18 - HR diagram

\section{Introduction}

This paper continues a series in which we present photometry of rich distant open clusters (Kaluzny 1994, Paper I; Kaluzny \& Rucinski 1995, Paper II). Berkeley $18=$ Be 18 $(l=163.3 \mathrm{deg}, b=+5.1 \mathrm{deg})$ is a faint cluster which has been identified by Setteducati \& Waever (1962). The color-magnitude diagram of this cluster was presented by Kaluzny et al. (1995). No detailed photometric study was reported so far for Be 18.

\section{Observations and data reduction}

Observations of Be 18 were collected during three observing runs at the Kitt Peak National Observatory. Table 1 gives essential information about these runs. Preliminary

Send offprint requests to: J. Kaluzny

* Visiting Astronomer, Kitt Peak National Observatory. KPNO is operated by AURA, Inc., under contract with the National Science Foundation.

1 Tables 3-5 are available in electronic form at the CDS, via ftp 130.79.128.5. processing of the data was done with the standard routines in the IRAF-CCDPROC package ${ }^{2}$. The flat-field frames were prepared by combining "dome flats" and exposures of the twilight sky. Such procedure was used while reducing data from all 3 runs.

Five overlapping sub-fields covering the central part of the cluster were observed during run \#1. We obtained four exposures for each sub-field: $120 \mathrm{~s}$ and $600 \mathrm{~s}$ exposures in the $V$-band, $240 \mathrm{~s}$ and $900 \mathrm{~s}$ exposures in the $B$-band. The sky was dark and clear during these observations. The seeing ranged from 1.1 to 1.3 arcsec. The instrumental photometry was extracted using DAOPHOT/ALLSTAR package (Stetson $1987,1991)$. A point spread function varying linearly with $X$ and $Y$ coordinates was adopted. 12 standard stars from the Landolt (1983) were observed on the night of October 3, 1991. In addition we observed twice 5 standards from the field around Ru 149 (Landolt 1992) and several standards from the so called "North consortium field" located in M92 (Stetson \& Harris 1988). The transformation equations adopted for the night of October 3, 1991 are:

$v=V+2.0635+0.025 \times(B-V)+0.16 \times(X-1.25)$
$b-v=0.231+0.912 \times(B-V)+0.096 \times(X-1.25)$

where $X$ is the airmass and the lower case letters refer to the instrumental magnitudes normalized to $1 \mathrm{~s}$ exposures. The standard deviations about magnitudes and colors for standard stars are 0.016 in $V$ and 0.019 in $(B-V)$. During our run the ST1K camera suffered from a charge transfer problem. The problem led to the formation of "shadowlike" traces on one side of very bright stars present in the frame, and affected photometry of some faint stars located in vicinity of the bright objects. The color-magnitude diagram (CMD) of Be 18 based on observations collected during run \#1 is shown in Fig. 1.

2 IRAF is distributed by the National Optical Astronomical Observatories, operated by AURA, Inc., under contract with the National Science Foundation. 
Table 1. Summary of observations of Be 18

\begin{tabular}{|c|c|c|c|c|c|c|}
\hline Run & $\begin{array}{c}\text { Date } \\
\text { UT }\end{array}$ & Telescope & Detector & $\begin{array}{l}\text { Scale } \\
" / \text { pix }\end{array}$ & $\begin{array}{c}\text { Field } \\
, 2\end{array}$ & Filters \\
\hline 1 & 1991 Oct. 3,4 & $2.1-\mathrm{m}$ & ST1K & 0.26 & $4.3 \times 4.3$ & $B V$ \\
\hline 2 & 1992 Oct. 19,20 & $0.9-\mathrm{m}$ & $\mathrm{T} 2 \mathrm{KA}$ & 0.68 & $23.0 \times 23.0$ & $B V I$ \\
\hline 3 & 1992 Oct. 29 & $2.1-\mathrm{m}$ & $\mathrm{T} 1 \mathrm{KA}$ & 0.30 & $5.1 \times 5.1$ & $B V I$ \\
\hline
\end{tabular}

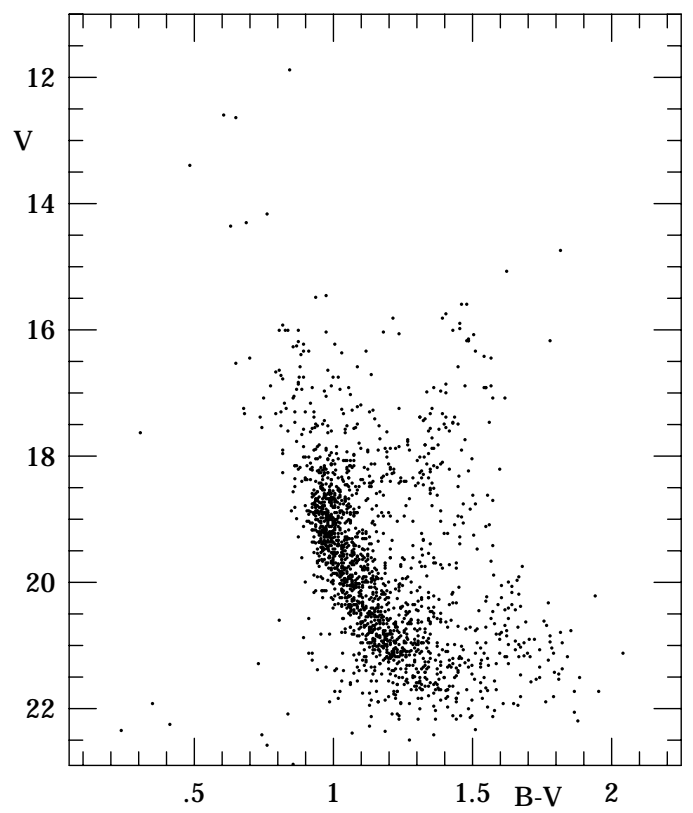

Fig. 1. $V$ vs. $B-V$ CMD for stars from the Be 18 region based on photometry obtained with the KPNO 2.1-m telescope + ST1K camera in October 1991

One field centered on the cluster center was observed during run \#2. During this run, the 0.9 -m telescope produced images with strong positional variability of the PSF. Stellar images were particularly poor for the first 500 columns of the chip. This section of frames was not used for photometry. About half of the frames were obtained with the telescope pointing offset by 500 pixels along rows. Hence, the effectively observed field had a size equivalent to the full field of view of the T2KA camera. A total of six images were obtained for each of $B V I$ filters. One short exposure $(180 \mathrm{~s}, 120 \mathrm{~s}$ and $70 \mathrm{~s}$ for $B, V$ and $I$ filters, respectively) and a pair of long exposures (900 s, $600 \mathrm{~s}$ and $420 \mathrm{~s}$, for $B, V$ and $I$ filters, respectively) were obtained for each filter and for each pointing. The seeing ranged from 1.65 arcsec to 2.45 arcsec. Moreover, on the night of October 19 the sky was covered with thin cirrus clouds. The the point spread function varying quadratically with $X$ and $Y$ coordinates was used to extract the profile photometry. The night of October 20 was photometric and the data obtained on that night were used to establish a transformation from the instrumental to the standard sys- tem. See Kaluzny \& Rucinski (1995) for details about the calibration procedure. The derived CMD's are presented in Fig. 2.

One field covering the central part of Be 18 was observed during run \#3. This run was plagued by bad weather. Some usefull data could be collected only during the last few hours of the last night of the run. Therefore, only a few exposures of the cluster were obtained. The log of observations is given in Table 2. Note that only one, relatively short exposure, was obtained in the $B$-band. The reduction procedure and details about transformation from the instrumental to the standard system were described in Kaluzny (1995). The derived CMD's are presented in Fig. 3.

The three data sets described above were calibrated independently of each other. The zero points of the $B V I$ photometry derived during runs \#2 and \#3 are in a good agreement. The largest systematic differences do not exceed $0.015 \mathrm{mag}$. The $B-V$ colors based on the data from run \#1 are systematicaly bluer by about 0.035 in comparison with colors based on runs \#2 and \#3.

Table 2. Log of Be 18 observations collected during run \#3

\begin{tabular}{lrcc}
\hline Filter & $\begin{array}{r}T_{\exp } \\
\text { sec }\end{array}$ & Airmass & $\begin{array}{c}\text { Seeing } \\
\operatorname{arcsec}\end{array}$ \\
\hline$B$ & 120 & 1.135 & 1.75 \\
$V$ & 90 & 1.139 & 1.66 \\
$V$ & 600 & 1.144 & 1.65 \\
$I$ & 60 & 1.158 & 1.67 \\
$I$ & 600 & 1.185 & 1.62 \\
\hline
\end{tabular}

\section{Radial extension}

Setteducati \& Weaver (1962) estimated the angular diameter of Be 18 at 20 arcmin. The cluster is located relatively close to the plane of the Milky Way and therefore its field is strongly contaminated by the background/foreground stars. To study the radial extension and richness of Be 18 we use data obtained with the $0.9-\mathrm{m}$ telescope. In Fig. 4 we show the observed luminosity function for stars with the available $V$-band photometry. This figure shows 


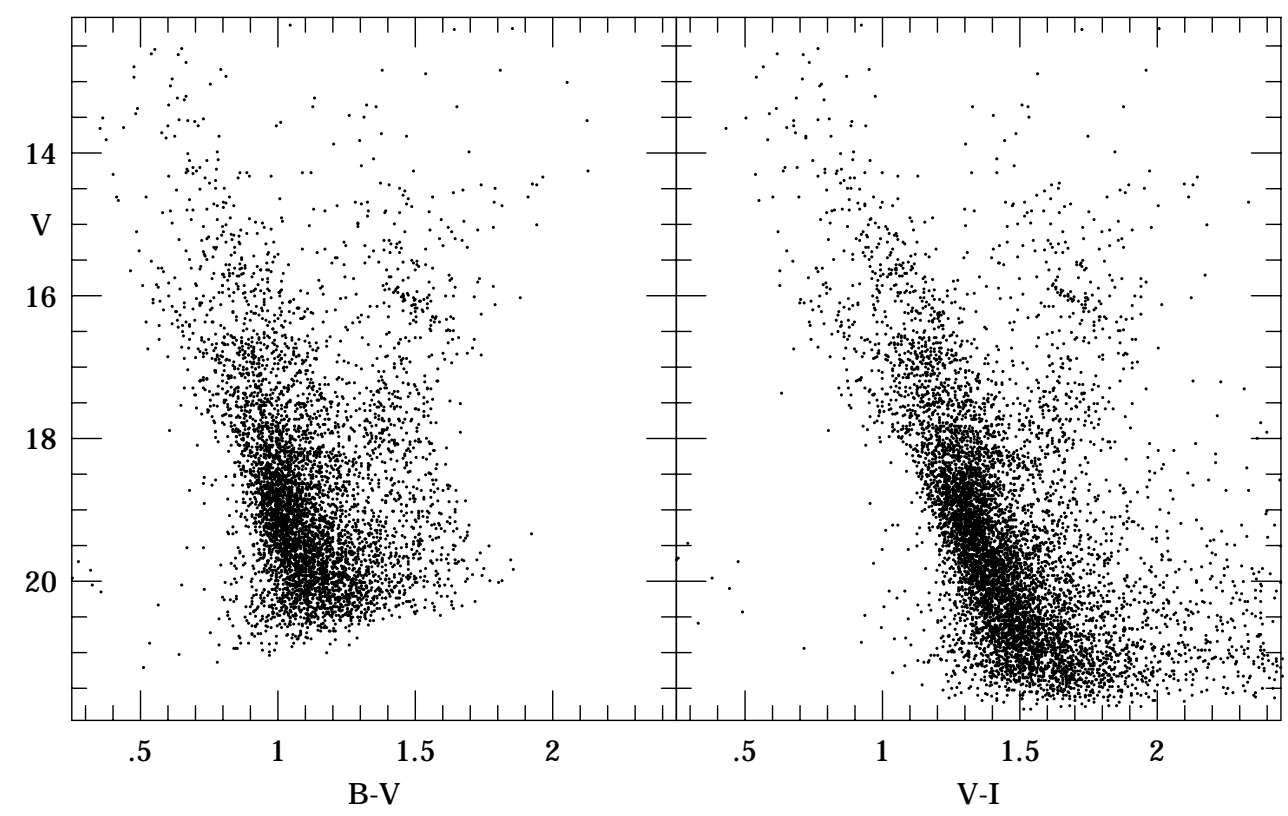

Fig. 2. $V$ vs. $B-V$ and $V$ vs. $V-I$ CMD's for stars from the Be 18 region based on photometry obtained with the KPNO 0.9-m telescope

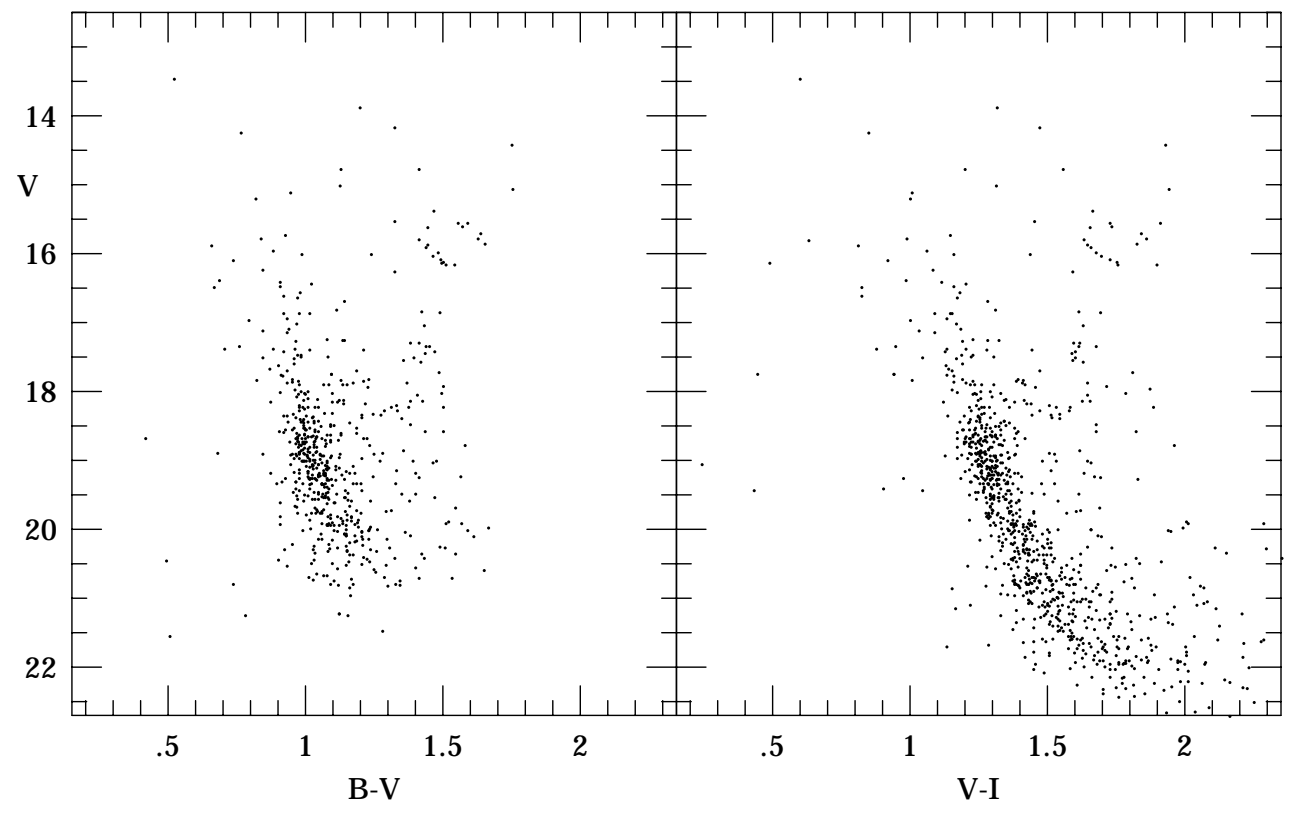

Fig. 3. $V$ vs. $B-V$ and $V$ vs. $V-I$ CMD's for stars from the Be 18 region based on photometry obtained with the KPNO 2.1-m telescope + T1KA camera in October 1992

that our photometry becomes significantly incomplete for $V>21.0$. On the other hand, the upper main-sequence of the cluster terminates at $V \approx 18.0$ (see Figs. 1-3). Consequently, we limited our attention to stars with $18.0<V<21.0$ while studying the radial extend of Be 18. The cluster center was determined at $(X, Y)=(1188,1036)$, where rectangular coordinates are given in pixels (as we describe in Appendix A all photometric data used in this paper are available in electronic form from editors of A\&A). The average stellar density was calculated in successive $47.6 \operatorname{arcsec}$ (70 pixels) wide annuli around the cluster center. Figure 5 shows the stellar surface density as a function of distance from the cluster center. The density profile flattens at radius $r \approx 800$ arcsec. Adopting $r=809$ arcsec for the cluster radius we derived $1.9010^{-3} \pm 0.1010^{-3}$ stars/ $\operatorname{arcsec}^{2}$ for the average surface density of the field stars. Adopting the above listed parameters we may estimate that about 4060 stars 


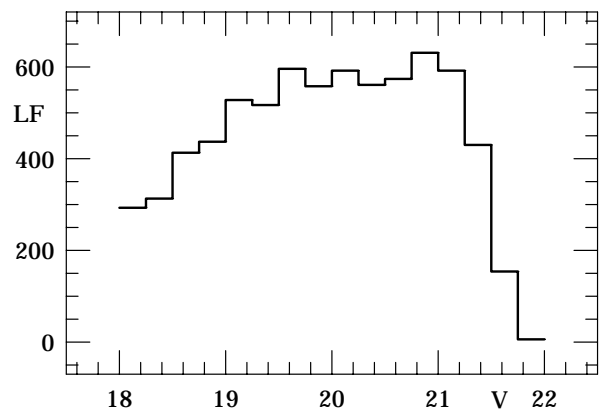

Fig. 4. The observed luminosity function for the field of Be 18 observed with the KPNO 0.9-m telescope. $L F$ is the number of stars in intervals 0.25 mag wide

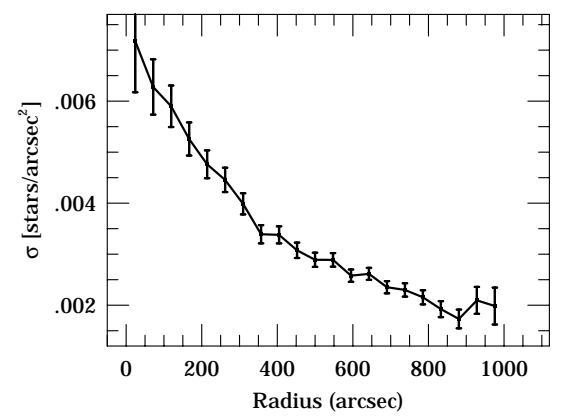

Fig. 5. Star density as a function of radial distance from the center of Be 18 for objects with $18<V<21.0$

with $18.0<V<21.0$ populate an upper main-sequence of the cluster. This estimate is in fact just a conservative lower limit. First of all we made no correction for the incompleteness of the photometry. Moreover, open clusters often possess extended coronas harboring a significant fraction of their member stars. Considering the number of upper-main sequence stars possessed by Be 18 we may conclude that it is one of the richest objects, if not the richest, in the whole sample of know old open clusters.

\section{Age, reddening and distance}

Even rough examination of CMDs presented in Figs. 1-3 leads to the conclusion that $\mathrm{Be} 18$ is an old open cluster. Its main-sequence terminates at $V \approx 18.0$ and the group of stars located at $V \approx 16.0$ and $B-V \approx 1.5$ can be identified with the cluster red giant branch. The data obtained during run \#3 were used to produce a CMD statistically cleaned from the field stars. We isolated two groups of stars from these data: those lying within radius $R=415$ arcsec from the cluster center, and those lying in an outer ring, between 619 and 809 arcsec. The inner circle and the observed part of an outer ring cover equal areas on the sky. The $V$ vs. $V-I$ CMDs for both selected subfields are presented in Fig. 6. As we demonstrated above, the radius of Be 18 is about 800 arcsec. Therefore the CMD for the outer ring contains some clus- ter members. Nevertheless the field stars are dominating group in this sample (see Fig. 5). Therefore the outer ring served as a "comparison field". For each star from this field a nearest match in the CMD of the inner ring was located. Subsequently, a pair of stars with the lowest separation was removed from both CMDs. This procedure was continued as long as it was impossible to locate pairs with a separation in magnitude $\delta V<0.5$ and a separation in color $\delta(V-I)<0.25$. The resulting "cleaned" CMD for the inner region of $\mathrm{Be} 18$ is shown in Fig. 7. It shows a well populated subgiant branch and a rich clump of helium burning giants. A group of stars forming bright extension of the cluster main-sequence contains candidates for blue/yellow stragglers. The CMD shown in Fig. 7 contains about 25 horizontal branch stars. The only known old open cluster with a comparably well populated horizontal branch is NGC 6791 possessing 22 stars in its red giant clump (Kaluzny \& Udalski 1992). The CMD for the whole field of Be 18 (see Fig. 2) contains about 35 candidates for the helium burning giants. Examination of Fig. $6 \mathrm{~b}$ shows that field stars give negligible contribution to this region of the cluster CMD.

The presented data are not sufficient for an accurate determination of such parameters of the cluster as its age, distance and metallicity. We can obtain only some limits on these parameters. Ages of old open clusters can be estimated with a good accuracy using the $\Delta V$ parameter, where $\Delta V$ is the magnitude difference between the turnoff and the clump of red giants. $\Delta V$ depends very weakly on the cluster metallicity and is almost independent of the reddening (e.g. Cassisi et al. 1994; Carraro \& Chiosi 1994). We note that slightly different working definitions of $\Delta V$ parameter are used by different groups (e.g. Phelps et al. 1994 vs. Paper I). Using definition from Paper I we get for Be $18 \Delta V \approx 1.9$. Based on the data published by Montgomery et al. (1994) we obtained $\Delta V=2.06$ for the well studied open cluster M 67. It may be concluded that Be 18 is slightly younger than M 67 . Current estimates of the age of M 67 group around 5 Gyr (Carraro \& Chiosi 1994; Hobbs \& Thorburn 1991; Nissen et al. 1987). Carraro and Chiosi derived the following relation between age, metallicity and $\Delta V$ :

$\log \left(t_{9}\right)=0.45 \times \Delta V+0.08 \times[\mathrm{Fe} / \mathrm{H}]+8.59$

where $t_{9}$ is age in billions of years. Assuming that M 67 and Be 18 have similar metallicities we arrive at the conclusion that the age of the later is about 4.3 Gyr. Being a distant cluster located in the galactic anticenter Be 18 has probably lower metallicity than M 67 . In such a case the age of Be 18 would be lower than $4.3 \mathrm{Gyr}$.

According to photometry published by Montgomery et al. (1994) the turnoff of M 67 occurs at $(B-V)=0.56$ which corresponds to $(B-V)_{0}=0.51$ if we adopt $E(B-V)=0.05$ for this cluster. The turnoff of Be 18 is observed at $(B-V) \approx 0.97$. Assuming for a moment 


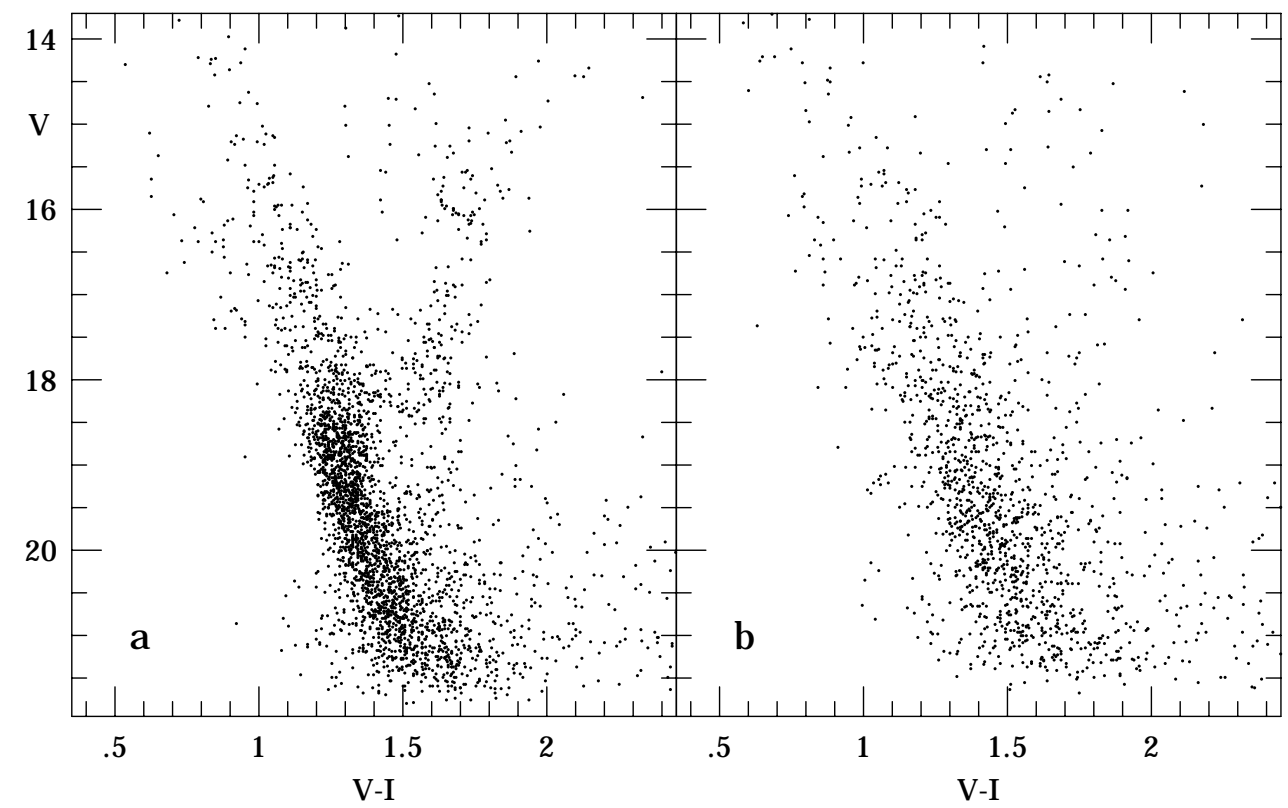

Fig. 6. The CMD's for the field covering central part of Be 18 (left) and for the "comparison" field (right)

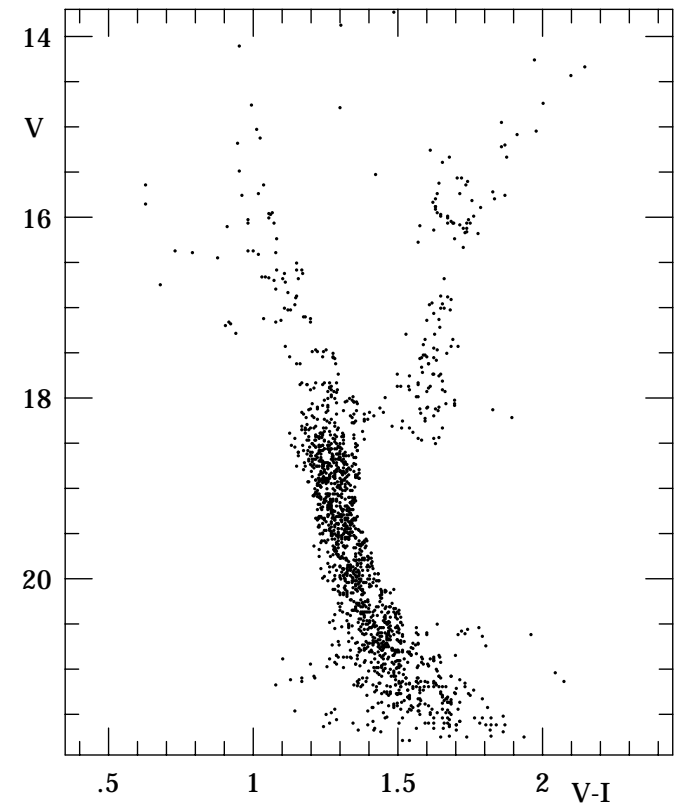

Fig. 7. Field-star corrected CMD for the central part of Be 18

that Be 18 and M 67 have the same age and metallicity we arrive at the conclusion that the reddening of the former cluster is $E(B-V) \approx 0.97-0.51=0.46$. In fact Be 18 is probably younger and metal poorer (note its location in the galactic anticenter) than M 67. Therefore, the turnoff of Be 18 is probably bluer than the turnoff of M 67 . In such a case $E(B-V)=0.46$ can be treated just as a conservative lower limit on the reddening of Be 18. A similar result can be obtained based on the analysis of the $V$ vs. $V-I$ CMD of the cluster.
For clusters with the solar metallicity the lower boundary of the red giant clump remains around $M_{V}^{\text {clump }} \approx 0.8$ for the range of ages $0.5-5 \mathrm{Gyr}$ (e.g. Cassisi et al. 1994). For Be 18 the faint end of the red horizontal branch is observed at $V \approx 16.09$. Hence, for the assumed solar metallicity of the cluster, we can estimate its apparent distance modulus at $(m-M)_{V} \approx 16.1-0.8=15.3$. Furthermore, adopting $E(B-V)=0.46$, we obtain $D=5.8 \mathrm{kpc}$ for the cluster distance. As it was noted above, the metallicity of Be 18 is likely to be lower than solar. This implies a higher reddening of the cluster but at the same time it leads to a higher value of $(M-m)_{V}\left(M_{V}^{\text {clump }}\right.$ becomes brighter for lower values of metallicity). Consequently, the above given estimate of the cluster distance is relatively insensitive to the adopted metallicity of the Be 18 . The galactocentric distance of the cluster is about $12.4 \mathrm{kpc}$ and its height above the galactic plane is about $520 \mathrm{pc}$. Concluding this section we note a large linear extension of Be 18. For the adopted distance of $5.4 \mathrm{kpc}$ and the angular size of about 26 arcmin we derive $44 \mathrm{pc}$ for the linear diameter of the cluster.

\subsection{The horizontal branch}

An interesting feature of the CMD of Be 18 is thre morphology of its horizontal branch. This branch is tilted and shows a relatively large extension - both verticaly and horizontaly - in comparison with other well studied old open cluster like M 67 or NGC 6791. Its width reaches 0.14 in the $B-V$ color and 0.12 in $V-I$. Particularly well defined horizontal branch is visible on the $V$ vs. $(V-I)$ CMD shown in Fig. 3. We note that the current models do not reproduce the observed extension of the red 
horizontal branch of Be 18. Another interesting feature which can be noted in Figs. 1-3 and Fig. 6 is a presence of an apparent sequence of stars located about 0.5 magnitude above the horizontal branch.

\section{Discussion and conclusions}

Detailed photometric study of Be 18 is difficult due to significant contamination of its field by the foreground stars. The faintness of the cluster is another complicating factor. Photometric data presented above show that Be 18 is a very rich old open cluster. The cluster age is similar to, or only slightly lower than the age of M 67 . The distance of Be 18 was estimated at $5.8 \mathrm{kpc}$ and its reddening is probably higher than $E(B-V)=0.46$. Independent spectroscopic determination of the metallicity of the cluster would allow more precise estimate of its parameters.

Acknowledgements. This work was supported by the Polish Committee of Scientific Research through grant 2P03D008.08 and by NSF grants AST 93-13620 and AST 92-16494 to Dr. Bohdan Paczyński. It is a pleasure to thank Dr. Bohdan Paczyński for his hospitality during the author's visit to Princeton.

\section{Appendix A}

Tables containing photometry presented in this paper are published by Astronomy and Astrophysics at the Centre de Données de Strasbourg. See the Editorial in A\&A 1993, Vol. 280, page E1.

\section{References}

Carraro G., Chiosi C., 1994, A\&A 287, 761

Cassisi S., Castellani V., Straniero O., 1994, A\&A 282, 753

Hobbs L.M., Thorburn J.A., 1991, AJ 102, 1070

Kaluzny J., 1994, A\&AS 108, 151 (Paper I)

Kaluzny J., 1995, Acta Astron. 44, 247

Kaluzny J., Udalski A., 1992, Acta Astron. 42, 29

Kaluzny J., Rucinski S.M., 1995, A\&AS 114, 1 (Paper II)

Kaluzny J., Krzemiński W., Mazur B., 1995, in "The formation of the Milky Way", Alfaro E.J., Delgado A.J., (eds). Cambridge Univ. Press, p. 185

Landolt A.U., 1983, AJ 88, 434

Landolt A.U., 1992, AJ 104, 340

Montgomery K.A., Janes K.A., Phelps R.L., 1994, AJ 108, 585

Nissen P.E., Twarog B.A., Crawford D.L., 1987, AJ 93, 634

Phelps R.L., Janes K.A., Montgomery K.A., 1994, AJ 107, 1079

Setteducati A.F., Waever M.F., 1962, Newly Found Stellar Clusters, published by Radio. Astr. Lab. Univ. of California, Berkeley

Stetson P.B., 1987, PASP 99, 191

Stetson P.B., 1991, in "Astrophysical Data Analysis Software and Systems I", ASP Conf. Ser. Vol. 25, Worrall D.M., Biemersderfer C. \& Barnes J. (eds.) p. 297

Stetson P.B., Harris W.E., 1988, AJ 96, 909 